\title{
OTHONOMICS
}

Revista de economía, empresa y sociedad

Dossier "Claves para entender el turismo de hoy"

UN GRAN POTENCIAL DE DESARROLLO

\section{Una aproximación a la investigación en responsabilidad social corporativa en el sector turístico}

\section{Lluís Garay Tamajón}

Profesor de los Estudios de Economía y Empresa (UOC)

RESUMEN La literatura sobre responsabilidad social corporativa (RSC) en el sector turístico surge en gran medida del paradigma del turismo sostenible, de donde ha extraído el interés por equilibrar los impactos de la empresa turística en los ejes ambiental, social y corporativo. Los dos temas que más referencias han generado son el business case o la posible relación entre su introducción y la mejora de los resultados de la empresa, y la relacionada con el compromiso con los stakeholders. Aparte, la literatura se ha preocupado por otros temas como la tipología de prácticas implementadas o la consecución de otro tipo de resultados a partir de estas. En todos estos campos existe todavía un gran potencial de desarrollo, a la vez que también emergen nuevos temas.

PALABRAS CLAVE responsabilidad social corporativa, turismo sostenible, business case; stakeholders; prácticas de RSC; resultados de la RSC

\section{An approach to researching corporate social responsibility in the tourism sector}

\begin{abstract}
Publications on Corporate Social Responsibility (CSR) in the tourism sector stem from the paradigm of sustainable tourism, in which there has been growing interest in relation to balancing the impacts of the tourism industry in terms of the key environmental, social and corporate issues. The two themes that have generated most references are, firstly, the business case or the possible relationship between its introduction and corporate financial performance, and secondly, commitments to stakeholders. Apart from these themes related to the drivers, publications have also focused on topics such as the types of practices implemented and other types of results achieved by them. In all these fields there is still great potential for development, while new themes are also emerging.
\end{abstract}

KEYWORDS corporate social responsibility; sustainable tourism; business case; stakeholders; CSR practices; CSR results 


\section{Introducción. El paradigma de la sostenibilidad como origen}

Las importantes transformaciones operadas en el consumo turístico en los últimos años han forzado a la oferta a realizar un importante esfuerzo de adaptación. El impacto de las tecnologías de la información y la comunicación (TIC) es quizás uno de los ejemplos más estudiados, pero también existen importantes cambios relacionados con la conciencia del impacto de la actividad turística en los destinos. A su estudio y al del diálogo de destinos y empresas turísticas con los diversos grupos de interés (stakeholders en la terminología anglosajona) se dedica, desde hace décadas, la literatura en torno a la sostenibilidad turística. El concepto se desarrolló en línea con las reflexiones del conocido Informe Brundtland (WCED, 1987), que establecía un nuevo paradigma de desarrollo fundamentado en tres principios: (1) continuar produciendo riqueza para satisfacer las necesidades de la población mundial (pilar económico), (2) velar por la reducción de las desigualdades entre los pueblos del mundo (pilar social) y (3) no degradar el medio ambiente que heredarán las generaciones futuras (pilar medioambiental).

La traslación al análisis del sector se concretó en el desarrollo del concepto de turismo sostenible, que se movió de una etapa inicial donde primó su conceptualización a otra donde los estudios de caso fueron adquiriendo mayor importancia, inicialmente centrados en el destino turístico, y donde la perspectiva macro y más vinculada a la planificación ha sido más prolíica (Ruhanen, Weiler y otros, 2015). Algunos autores (Coles, Fenclova y otros, 2013) indican que esto empezó a cambiar desde que la Declaración de Ciudad del Cabo en 2002 señaló la necesidad de que los sistemas de producción fueran más responsables, apelando directamente a las organizaciones. La respuesta en el análisis de la empresa turística vino de la mano de un paradigma de largo recorrido en el análisis empresarial, el de la responsabilidad social corporativa (RSC), que ha evolucionado a la par que la propia concepción de empresa, pero que ha adquirido mayor peso en los últimos lustros.

\section{Del concepto general a su aplicación al ámbito turístico}

El concepto de RSC se originó en el mundo de la empresa en los años treinta en relación con la necesidad de que los directivos actuaran más allá de sus expectativas de ganancias económicas. Con todo, no sería hasta los setenta cuando empezó a tener cierta resonancia teórica con la discusión sobre cuáles eran realmente las responsabilidades de las empresas más allá de su actividad (Friedman, 1970; Freeman, 1984). De este debate surgió la idea de que la RSC representaba, por un lado, una contribución de carácter voluntario (en línea con el paradigma neoliberal) a la mejora del medio ambiente, la sociedad y la economía por parte de las empresas, fuera por motivos altruistas y/o con el objetivo de mejorar su situación competitiva. De aquí surgió, por un lado, el denominado business case, donde la implementación de la RSC parte de una motivación economicista que busca encontrar una relación entre esta y los resultados de la empresa, especialmente los financieros (Carroll y Shabana, 2010), sin que haya habido consenso al respecto (Lee, 2008), lo que se relaciona también con las dificultades de medida, la falta de especificaciones o la cultura empresarial en determinados casos. Por el otro, siguiendo las ideas de Freeman (1984), también se interpretó como una respuesta que va más allá del reporte a los accionistas, teniendo en cuenta las expectativas de otros grupos de interés o stakeholders, desde los internos (accionistas, trabajadores) a los externos (consumidores, proveedores, socios, llegando a la administración, otras organizaciones e incluso a la sociedad, el medioambiente o a los intereses de las generaciones venideras).

De hecho, la misma definición de lo que es o debería ser RSC en el sector turístico ha sido también objeto de discusión y ha evolucionado en el tiempo; ha predominado el análisis de subsectores como el del alojamiento y la aviación (Coles, Fenclova y otros, 2013) y con una menor atención al sector de la intermediación (Garay, Gomis y otros, 2017). En todo caso parece que todavía nos encontramos en un contexto en el que la literatura acerca de la RSC en la empresa turística ha sido escasa en comparación con otros sectores (Bohdanowicz y Zientara, 2008), aunque creciente, en los últimos años (Kang, Lee y otros, 2010), utilizando diferentes metodologías y con especial atención a las dos temáticas resaltadas anteriormente. Más allá de estos grandes temas, aparecen nuevos temas relacionados como la ética empresarial (Yaman y Gurel, 2006), el turismo en favor del alivio de la pobreza 
(Schilcher, 2007), la certificación de la sostenibilidad (Font y Harris, 2004), la gestión ambiental (Ayuso, 2006) o el marketing relacionado (Dief y Font, 2010).

\section{Principales temáticas}

\subsection{Motivaciones}

\section{Business case}

Una de las grandes áreas de discusión en la literatura sobre RSC en el sector turístico es la de la consideración sobre las motivaciones para implementarla. En este sentido, de la misma manera que sucedía en la literatura genérica, destaca el denominado business case, o la motivación para introducirla porque se relaciona positivamente con los resultados. Especialmente a través de la denominada visión basada en los recursos y las competencias (resource based view), Inoue, Kent y otros (2011) han sugerido que la implementación de la RSC en el sector puede venir motivada por la posibilidad de originar recursos y competencias, distintivos y valiosos, que podrán proporcionar a la empresa una futura ventaja competitiva basada en la diferenciación. Más allá de los resultados financieros, otros estudios (Deery, Jago y otros, 2007) han encontrado relaciones positivas en cuanto al poder reclutar y retener a los empleados, así como a ganancias de productividad. En muchos casos, el business case se relaciona directamente con las motivaciones vinculadas no solo a la reducción de costes o la mejora de los resultados, sino que, indirectamente, a través de la mejora de la imagen de la empresa o los aspectos vinculados al marketing (Tsai, Hsu y otros, 2010). Además, en el contexto de las pequeñas y medianas empresas (pymes), donde los valores de los empresarios son básicos en el momento de introducir la RSC, también se puede encontrar el pragmatismo económico derivado de la necesidad de mantener cierto posicionamiento en el mercado o de la presión o influencia de determinados stakeholders (Murillo y Lozano, 2006), relacionado, en gran medida, con el siguiente gran tema.

\section{Compromiso con los stakeholders}

La anterior aproximación, aunque válida para un amplio conjunto de empresas, parece dejar sin explicar gran parte del comportamiento responsable y el mismo concepto de RSC que se relaciona desde hace ya años con la teoría de los stakeholders o grupos de interés, y la necesidad de legitimar las operaciones de la empresa turística frente a los mismos y/o frente a la sociedad en general (Ayuso, 2006; Inoue, Kent y otros, 2011). Al respecto de este compromiso, se ha hablado especialmente de su posible aplicación para el caso de las pymes, aunque parece claro que los grupos de interés prioritarios difieren de los de las grandes corporaciones, como ocurre en el caso de los empleados, con los que existe una mayor relación y necesidad de compromiso (Murillo y Lozano, 2006) o la comunidad donde se opera, con la que la relación también suele ser mucho más estrecha y personal (Perrini, 2006). En la literatura se desprende un especial interés por comprender la relación con determinados grupos de interés con los que las empresas turísticas tienen mayor relación: turistas, residentes en el destino, emprendedores y administración local (Dodds y Kuehnel, 2010), que pueden tener intereses notablemente diferentes, lo que da lugar, en ocasiones, a tensiones y conflictos que la empresa debe gestionar (Andriotis, 2005).

\section{Valores, hábitos y estilos de vida}

Un tercer marco explicativo sugiere que parte del comportamiento en favor de la RSC observada, especialmente en las pymes, se explica mejor a través de los valores, hábitos y estilos de vida de los propietarios y/o directivos que de determinadas acciones conscientes. Según la literatura, en las pymes, los valores de los propietarios (morales, religiosos, personales, comunitarios, etc.) son un factor clave en la introducción de la RSC (Spence, 2007), y es especialmente importante, también, el carácter emprendedor que tienen muchos de estos (necesidad de alcanzar metas, tolerancia a la ambigüedad, mayor desarrollo moral). De la consideración conjunta de tales 
elementos (importancia de los valores y carácter emprendedor) surge el concepto de Lifestyle Entreperneur, que se ha planteado frecuentemente al hablar de la responsabilidad y otros aspectos en las pymes (Shaw, Williams y otros, 2004); se entiende como aquel propietario que pone en balance la generación de ingresos con la preservación de su estilo de vida.

\subsection{Prácticas y resultados}

\section{Prácticas}

Los motivos mencionados anteriormente explican en gran parte las prácticas reportadas, clasificadas como organizacionales y operacionales (Dief y Font, 2010); también a partir de los tradicionales ejes ambiental, social y económico (Inoue, Kent y otros, 2011). En el caso de las prácticas organizacionales, cabe decir que son relevantes para el desarrollo y la implementación de sistemas de gestión de RSC que ayudan a las empresas a identificar y administrar los elementos relacionados con la responsabilidad y sus consecuencias de manera integral y coherente (Bonilla Priego, Nájera y otros, 2011). En todo caso, sobre todo se han identificado prácticas operacionales que implican modificaciones en la producción y en los sistemas de operaciones, y que son específicas de este sector (Carmona-Moreno, Céspedes-Lorente y otros, 2004), especialmente en el caso de las pymes (Tzschentke, Kirk y otros, 2004). La caracterización de estas prácticas operacionales es amplia y a veces ambigua, pero parece dominada por la presencia de acciones ambientales para reducir los costes de operación, minimizar el consumo de recursos y/o introducir compras «verdes» (Tzschentke, Kirk y otros, 2004; Ayuso, 2006; Bohdanowicz y Zientara, 2008). También resultan interesantes algunas aproximaciones que observan una posible falta de comunicación de algunas de las medidas fruto del miedo de algunas empresas a un posible mal uso de esa información, u otras, (Dodds y Kuehnel, 2010) que apuntan a que los empresarios consideran la implementación de menor importancia y que quizás seria necesaria una mayor regulación por parte de los Gobiernos para impulsarla (Bohdanowicz y Zientara, 2008).

\section{Resultados}

Siguiendo el paradigma del business case, los primeros estudios sobre RSC para el sector turístico, centrados en el sector hotelero, ya confirmaban la introducción de medidas básicas encaminadas a la consecución de ganancias financieras (Kirk, 1995). En todo caso, como se comentó antes, los metaestudios al respecto no son concluyentes, aunque algunas referencias más innovadoras han observado que en realidad esta relación puede tener la forma de una $U$ invertida (Kang, Lee y otros, 2010), donde existe un nivel óptimo de introducción de medidas de RSC que contribuye a la mejora de los resultados; la actuación más allá de esto solo se explica en función de motivos no económicos. Sin embargo, para el caso de las pymes, se ha de seguir haciendo hincapié en la explicación de la ventaja competitiva potencial de la aplicación de las teorías del capital social (Fuller y Tian, 2006), que en gran medida también se relacionan con las teorías sobre los stakeho/ders y que, de alguna manera, desmienten la teoría de que la responsabilidad empresarial sea un «bien de lujo».

\section{Conclusiones}

Existe todavía un gran potencial para que los análisis sobre la RSC en el sector superen una primera etapa de racionalización del concepto. En el caso del business case porque existe se puede extender este tipo de análisis a otros aspectos tan interesantes como la mejora de la satisfacción, la productividad o la reputación. En el de los stakeholders porque es posible dar voz a determinados grupos de interés, especialmente internos, capaces de aportar información de gran interés a la vez que se puede impulsar, también, el análisis de la comunicación con estos grupos de interés. También existe un considerable potencial en cuanto al estudio de las fuentes de información y la comunicación de la RSC (Coles, Fenclova y otros, 2013). En este sentido, mejorar la información 
en responsabilidad y saber cómo esta se transforma en conocimiento (Font, Garay y otros, 2016) parece todo un reto para averiguar cómo transformar las buenas prácticas reportadas en una mejora de los resultados.

Por último, también existen retos que tienen que ver con el análisis de la relación entre la RSC y los resultados, ya que, aunque muchos estudios hayan partido de la asunción de una fuerte relación entre estos elementos, esta todavía se debe por probar de manera genérica. Como diferentes autores señalan (Lee, 2008; Kang, Lee y otros, 2010; Inoue, Kent y otros, 2011) falta mejorar la obtención de información, o la credibilidad de los efectos observados, así como disponer de más datos a lo largo del tiempo y en diferentes subsectores turísticos. También hay que tener en cuenta la falta de más estudios en determinadas regiones, así como en algunos subsectores como el de la intermediación, y especialmente en el ámbito de los turoperadores, empresas con una gran capacidad de arrastre en el sector; también falta un mayor número de estudios transnacionales, dado que precisamente el turismo es uno de los grandes protagonistas de la globalización.

\section{Referencias bibliográficas}

ANDRIOTIS, K. (2005). "Community groups» perceptions of and preferences for tourism development: Evidence from Crete». Journal of Hospitality \& Tourism Research. Vol. 29, n. 1, págs. 67-90.

AYUSO, S. (2006). «Adoption of voluntary environmental tools for sustainable tourism: analysing the experience of Spanish hotels». Corporate social responsibility and environmental management. Vol. 13, n. ${ }^{\circ}$ 4, págs. 207-220.

BOHDANOWICZ, P.; ZIENTARA, P. (2008). «Corporate social responsibility in hospitality: Issues and implications. A case study of Scandic». Scandinavian Journal of Hospitality and Tourism. Vol. 8, n. 4, págs. 271-293.

BONILLA PRIEGO, M. J.; NÁJERA, J. J.; FONT, X. (2011). «Environmental management decision-making in certified hotels». Journal of Sustainable Tourism. Vol. 19, n. ${ }^{\circ}$ 3, págs. 361-381.

CARMONA-MORENO, E.; CÉSPEDES-LORENTE, J.; DE BURGOS-JIMÉNEZ, J. (2004). «Environmental strategies in Spanish hotels: contextual factors and performance». The Service Industries Journal. Vol. 24, n. ${ }^{\circ}$, págs. 101-130.

CARROLL, A. B.; SHABANA, K. M. (2010). «The business case for corporate social responsibility: A review of concepts, research and practice». International journal of management reviews. Vol. 12, n. ${ }^{\circ}$, págs. 85-105.

COLES, T.; FENCLOVA, E.; DINAN, C. (2013). «Tourism and corporate social responsibility: A critical review and research agenda». Tourism Management Perspectives. Vol. 6, págs. 122-141.

DEERY, M.; JAGO, L.; STEWART, M. (2007). "Corporate social responsibility within the hospitality industry». Tourism Review International. Vol. 11, n. ${ }^{\circ}$ 2, págs. 107-114.

DIEF, M. E.; FONT, X. (2010). "The determinants of hotels marketing managers' green marketing behaviour». Journal of Sustainable Tourism. Vol. 18, n. ${ }^{\circ} 2$, págs. 157-174.

DODDS, R.; KUEHNEL, J. (2010). «CSR among Canadian mass tour operators: good awareness but little action». International Journal of Contemporary Hospitality Management. Vol. 22, n. ${ }^{\circ}$ 2, págs. 221-244.

FONT, X.; GARAY, L.; JONES, S. (2016). «A Social Cognitive Theory of sustainability empathy». Annals of Tourism Research. Vol. 58, págs. 65-80.

FONT, X.; HARRIS, C. (2004). «Rethinking standards from green to sustainable». Annals of Tourism Research. Vol. 31, n. ${ }^{\circ}$ 4, págs. 986-1007.

FREEMAN, R. E. (1984). Strategic management: A stakeholder approach. New York: Pitman.

FRIEDMAN, M. (1970). «The social responsibility of business is to increase its profits». New York Times Magazine. Septiembre 13, págs. 122-124.

FULLER, T.; TIAN, Y. (2006). «Social and symbolic capital and responsible entrepreneurship: an empirical investigation of SME narratives». Journal of business Ethics. Vol. 67, n. ${ }^{\circ}$ 3, págs. 287-304.

GARAY, L.; GOMIS, J.; GONZÁLEZ, F. (2017). «Management, Altruism and Customer Focus as drivers of Corporate Social Responsibility in Tourism Intermediation». Tourism Analysis. Vol. 21, n. ${ }^{\circ} 3$.

INOUE, Y.; KENT, A.; LEE, S. (2011). "CSR and the bottom line: Analyzing the link between CSR and financial performance for professional teams». Journal of Sport Management. Vol. 25, n. ${ }^{\circ} 6$, págs. 531-549. 
KANG, K. H.; LEE, S.; HUH, C. (2010). «Impacts of positive and negative corporate social responsibility activities on company performance in the hospitality industry». International Journal of Hospitality Management. Vol. 29, n. ${ }^{\circ} 1$, págs. $72-82$

KIRK, D. (1995). «Environmental management in hotels». International Journal of Contemporary Hospitality Management. Vol. 7, n. ${ }^{\circ}$ 6, págs. 3-8.

LEE, M. D. P. (2008). «A review of the theories of corporate social responsibility: Its evolutionary path and the road ahead». International journal of management reviews. Vol. 10, n. ${ }^{\circ}$ 1, págs. 53-73.

MURILLO, D.; LOZANO, J. M. (2006). «SMEs and CSR: An approach to CSR in their own words». Journal of business Ethics. Vol. 67, n. ${ }^{\circ}$ 3, págs. 227-240.

PERRINI, F. (2006). "SMEs and CSR theory: Evidence and implications from an Italian perspective». Journal of business Ethics. Vol. 67, n. ${ }^{\circ}$ 3, págs. 305-316.

RUHANEN, L.; WEILER, B.; MOYLE, B. D.; y otros (2015). «Trends and patterns in sustainable tourism research: a 25-year bibliometric analysis». Journal of Sustainable Tourism. Vol. 23, n. ${ }^{\circ} 4$, págs. 517-535.

SCHILCHER, D. (2007). "Growth versus equity: The continuum of pro-poor tourism and neoliberal governance». Current Issues in Tourism. Vol. 10, n. ${ }^{\circ} 2-3$, págs. 166-193.

SHAW, G.; WILLIAMS, A.; THOMAS, R. (2004). «From lifestyle consumption to lifestyle production: changing patterns of tourism entrepreneurship». Small firms in tourism. Capítulo 7. págs. 99-113. Elsevier Science B. V.

SPENCE, L. J. (2007). "CSR and small business in a European policy context: the five "C" $s$ of CSR and small business research agenda 2007». Business and society review. Vol. 112, n. ${ }^{\circ}$ 4, págs. 533-552.

TSAI, W.-H.; HSU, J.-L.; CHEN, C.-H. y otros. (2010). «An integrated approach for selecting corporate social responsibility programs and costs evaluation in the international tourist hotel». International Journal of Hospitality Management. Vol. 29, n. ${ }^{\circ}$ 3, págs. 385-396.

TZSCHENTKE, N.; KIRK, D.; LYNCH, P. A. (2004). «Reasons for going green in serviced accommodation establishments». International Journal of Contemporary Hospitality Management. Vol. 16, n. ${ }^{\circ}$ 2, págs. 116-124.

WCED (1987). "Our common future». World Commission on Environment and Development Oxford University Press.

YAMAN, H. R.; GUREL, E. (2006). «Ethical ideologies of tourism marketers». Annals of Tourism Research. Vol. 33, n. ${ }^{\circ} 2$, págs. $470-489$.

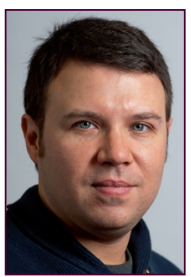

\section{Luís Garay Tamajón}

lgaray@uoc.edu

\section{Profesor de los Estudios de Economía y Empresa (UOC)}

Profesor de la Universitat Oberta de Catalunya (UOC) desde el año 2002, donde ha impartido asignaturas del ámbito de la economía del turismo, así como de la historia y el pensamiento económico. En la misma UOC ha sido director del Segundo Ciclo de ADE y del de Ciencias del Trabajo, así como del grado en Relaciones Laborales y Ocupación. Desde 2014 es director del máster universitario de Responsabilidad Social Corporativa de la misma universidad. Su investigación se inició con una tesis presentada en 2007 que supuso un estudio pionero sobre la historia del turismo en Cataluña, para continuar analizando diferentes productos turísticos. Desde 2010, cuando realizó una estancia posdoctoral en la Leeds Metropolitan University, su investigación se centra sobre todo en los motivos para implementar la responsabilidad social corporativa en la empresa turística, con especial atención al caso de las pequeñas y medianas empresas. 
Los textos publicados en esta revista están sujetas -salvo que se indique el contrario- a una licencia de Reconocimiento 3.0 España de Creative Commons. Podéis copiarlos, distribuirlos, comunicarlos públicamente y hacer obras derivadas siempre que reconozcáis los créditos de las obras (autoría, nombre de la revista, institución editora) de la manera especificada por los autores o por la revista. La licencia completa se puede consultar en http://creativecommons.org/licenses/by/3.0/es/deed.ca.

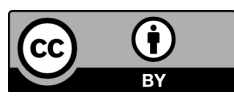

